\title{
Ferroresonance Phenomenon in CFE, its Origin and Effects
}

\author{
E. Martínez, G. Antonova, M. Olguín
}

\begin{abstract}
Technological improvements and energy exchange between and within electrical systems require more availability and reliability, and the use of communication channels and measuring devices helps to achieve this goal. Coupling Capacitor Voltage Transformers (CCVTs) traditionally have been essential for power systems communications using Power Line Carrier (PLC), and its design normally includes a protection to prevent ferroresonance phenomenon. However, CCVTs failures due to environmental conditions and their lack of accuracy for energy billing led some electrical companies like the Mexican company Comisión Federal de Electricidad (CFE) to gradual replacement of CCVT devices by the Inductive Voltage Transformers (IVT), which are installed now in busbars of electrical substations. In addition to satisfy communications PLCs were replaced by the optical fiber communications. As IVT devices do not have anti ferroresonance elements, ferroresonance phenomenon started to appear more frequently during operation maneuvers in several electrical substations. This paper presents background on CCVT explosions, patterns of behavior before failures, Phasor Measurement Unit (PMU) records for system effects, measurements and simulation of typical ferroresonance occurrences. Ferroresonance mitigation techniques and possible protection schemes are also discussed.
\end{abstract}

Keywords: ferroresonance, inductive voltage transformer, damping reactor, phasor measurement unit.

\section{INTRODUCTION}

$\mathrm{F}$ erroresonance or nonlinear resonance is a type of resonance in electric circuits which occurs when a circuit containing a non-linear inductance is fed from a source with series and parallel capacitance, and the circuit is subjected to a disturbance such as opening of a circuit breaker [1]. It can cause over voltages and over currents in an electrical power system and can pose a risk to transmission and distribution equipment and to operational personnel [2].

Ferroresonance should not be confused with linear resonance that occurs when inductive and capacitive reactances of a circuit are equal. In linear resonance the

E. Martínez is with Comisión Federal de Electricidad, Subdirección de Transmisión, Don Manuelito $N^{\circ} 32$ Col. Olivar de los Padres, D. F., México (e-mail: enrique.martinez03@cfe.gob.mx ).

G. Antonova is with ABB, Vancouver, Canada (e-mail: galina.s.antonova@ca.abb.com).

M. Olguín is with Comisión Federal de Electricidad, Subdirección de Transmisión. Don Manuelito $N^{\circ} 32$ Col. Olivar de los Padres, D. F., México (e-mail: miguel.olguin@cfe.gob.mx ).

Paper submitted to the International Conference on Power Systems Transients (IPST2013) in Vancouver, Canada July 18-20, 2013. current and voltage relationship is linear and frequency dependent. In the case of ferroresonance it is characterized by a sudden jump of voltage or current from one stable operating state to another one. The relationship between voltage and current depends not only on the frequency but also on a number of other factors such as the system voltage magnitude, initial magnetic flux condition of transformer iron core, the total loss in the ferroresonant circuit and the point on wave of initial switching [2].

The first work on ferroresonance was made by Joseph Bethenod in 1907, who described the phenomenon of ferroresonance simply as transformer resonance [3]. In 1920 French engineer Paul Boucherot first used the term "ferroresonance" to describe the phenomenon of two stable fundamental frequency operating points, coexisting in a series circuit containing a resistor, nonlinear inductor and a capacitor [4].

The ferroresonant circuit can be formed with a non-linear inductive ferromagnetic core such as a transformer, capacitance and a voltage or current source. In linear circuits, resonance occurs when the capacitive reactance is equal to the inductive reactance at the frequency of the power circuit. Unlike the linear resonance, the ferroresonance is not easy to predict due to the nonlinearity. Ferroresonant circuit is very sensitive to initial conditions: residual flux in the magnetic cores, switching and power losses of the circuit and load capacitances.

The ferroresonance is hazardous to the integrity of electrical equipment and to the operating personnel. High voltages or currents may overheat the transformer primary winding and can cause breakdown of insulation. The high voltage oscillations (temporary or sustained) may cause damage to the insulation of the equipment involved in the ferroresonant circuit.

Recently there have been several ferroresonance cases in the Mexican Electric Systems (MES). This paper presents background on the ferroresonance origin such as history and analysis of CCVT explosions, patterns of behavior before failures, Phasor Measurement Unit (PMU) records for system effects, as well as measurements and simulation of typical ferroresonance occurrences, ferroresonance mitigation techniques and possible protection schemes.

\section{FerroresonAnce Conditions, Modes AND EFFECTS}

As explained earlier, the ferroresonance can appear in series electric circuits containing a resistor, nonlinear inductor and a capacitor. Specific conditions, operating modes and effects of the ferroresonance are described below. Ferroresonance 
equivalent circuit is shown on Fig. 1, where $\mathrm{C}_{-} \mathrm{S}$ is series capacitance (typically, this is capacitance of an open breaker), C_p is parallel capacitance (typically, this is bus capacitance) and IVT, a non-linear inductance of IVT.

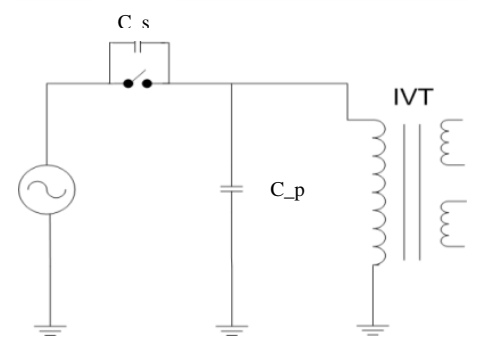

Fig. 1. Ferroresonance equivalent circuit.

\section{A. Ferroresonance conditions}

In the electrical distribution system ferroresonance typically includes a medium voltage electrical distribution network of transformers (inductive component) and power cables (capacitive component) [5], [6]. If such network has little or no resistive load connected and one phase of the applied voltage is interrupted, a ferroresonance can occur. If the remaining phases are not quickly disconnected, the phenomenon continues, and overvoltage can lead to the breakdown of insulation in the connected components resulting in their failure.

Ferroresonance phenomenon can be avoided by connecting a minimal resistive load to the transformer's secondary winding or by disconnecting the applied voltage by a 3-phase interrupting device such as a 3-pole circuit breaker [5].

Ferroresonance conditions include capacitors, saturable inductors and low energy losses. The capacitance is provided by circuit breakers (with grading capacitance), power transmission components (cable capacitance, busbar capacitance, coupling between lines and capacitor banks), insulating elements (isolation capacitance) and measurement equipment (capacitive transformers).

Saturable or non-linear inductance is provided by reactors, power transformers and inductive voltage transformers. In addition, systems with low resistance (transformers and circuits working in low-loss mode) increase the probability of ferroresonance occurrence [6].

\section{B. Ferroresonance modes}

There are four ferroresonance modes defined for the steady-state condition [7] :

- fundamental,

- sub harmonic,

- quasi-periodic, and

- Chaotic

In the fundamental mode the voltages and currents are periodic with a period $\mathrm{T}$ that is equal to the electrical network period and can contain a variety of harmonics, see Fig. 2(a).

In the sub harmonic mode the signals are periodic, with a period $n T$ that is equal to a multiple of the electrical network period. This mode is called sub harmonic $\mathrm{n}$ or harmonic $1 / \mathrm{n}$, where $n$ is usually an odd number, see Fig. 2(b).

In the quasi-periodic mode the signals are not periodic and the corresponding frequency spectrum is discontinuous, that means not all frequencies are present, see Fig. 2(c).

In the chaotic mode the signals are not periodic and the corresponding frequency spectrum is continuous, i.e. all frequencies are present, see Fig. 2(d).

The most common modes are fundamental and sub harmonic [8].

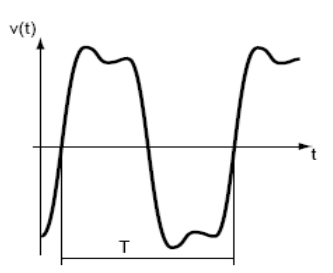

(a)

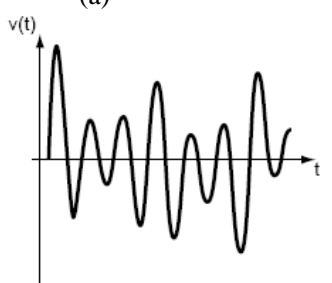

(c)

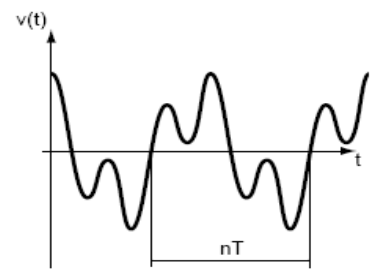

(b)

(d)

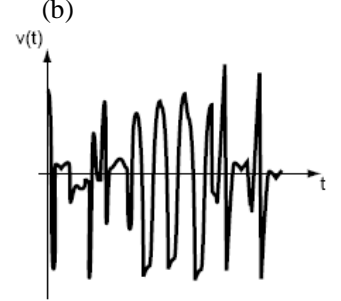

Fig. 2. Ferroresonance modes:

(a) fundamental, (b) sub harmonic, (c) quasi-periodic and (d) chaotic.

Small variations in the parameters values of the electrical system or a transient could cause a sudden change to a different stable state that can cause one of the four ferroresonance modes.

The parameters which may determine the ferroresonance mode are:

- coupling capacitance which determines the natural frequency of oscillation,

- amplitude of the voltage source, and

- resistive losses of the core

It should be noted that while the ferroresonance modes are defined for steady-state conditions, transient over voltages could occur several cycles after an event and can persist for several cycles, which can result in a serious damage to the electrical equipment involved.

\section{Ferroresonance effects}

Ferroresonance can cause the following hazardous effects in electrical power systems:

- sustained phase-to-phase and phase-to-ground over voltages

- sustained over currents

- sustained waveforms distortions of currents, voltages

- transformers heating up

- electrical equipment damage (thermal or due to insulation failure)

- loss of protective devices operation

- overheating of walls and metal parts of the transformer due to dispersion flux and high saturation of the core and internal parts of the transformer

- damage in surge arresters, surge suppressors and 
overvoltage protection devices due to overheating.

- transformer's life reduction due to overheating and dielectric stress [9].

\section{FERroresonANCE IN MEXICAN SYSTEM}

Mexican Electrical System owned and operated by the Comisión Federal de Electricidad (CFE) consists of three independent electric power systems:

- National Interconnected Power System (Sistema Interconectado Nacional),

- North Baja California Electric Power System (Sistema Eléctrico de Baja California Norte), and

- South Baja California Electric Power System (Sistema Eléctrico de Baja California Sur).

Voltage levels in CFE transmission and sub-transmission systems are 400, 230, 161, 138 and $115 \mathrm{KV}$. Until a decade ago, all these systems used PLC for communications. In addition to traditional voice and data communications service, PLC equipment supports more critical teleprotection applications. For the latter CCVT installations in phases of transmission lines are essential, these are illustrated in Fig. 3.

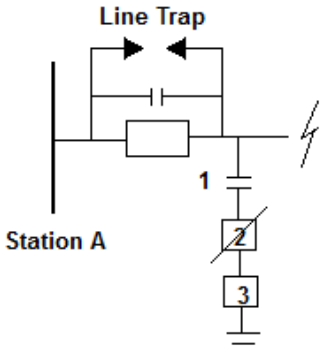

1 CCVT

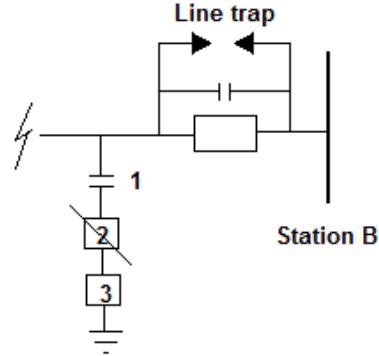

3 Transmitter / Receiver
2 Line Tuner

Fig. 3. Power Line Carrier diagram.

The CCVTs, however have been exploding causing loss of energy service, collateral damage to substation equipment and risk to operating personnel. Fault records analysis has shown that most of the explosions were due to defective design or manufacturing defects and extreme environmental conditions of the operating sites. CCVT failure statistics is given in Table I.

CCVT faults were analyzed using date, time, location, temperature and humidity during the explosion. To find a pattern, PMU records were used prior, during and after the explosion. It was found that an unbalance caused by a degradation of the damaged phase occurred 45 to 90 minutes before the CCVT explosion, see Fig. 4.

Based on this analysis, it would be possible to implement alarms and controls to open circuits upon detection of phase voltage imbalance, and replace the CCVT before explosion to avoid power loss and collateral damage to the nearby personnel and equipment. The CCVT failures and increased accuracy requirements for the energy billing were the determining factors for re-placing CCVTs by the IVTs. In parallel CFE was developing a fiber-optical network, to gradually replace the communication systems as well.
TABLE I

CCVT FAILURES IN CFE SYSTEM

\begin{tabular}{|c|c|c|c|c|}
\hline YEAR & $400 \mathrm{KV}$ & $230 \mathrm{KV}$ & $115 \mathrm{KV}$ & TOTAL \\
\hline 2010 & 1 & 4 & 2 & 7 \\
\hline 2009 & 11 & 5 & 2 & 18 \\
\hline 2008 & 3 & 4 & 0 & 7 \\
\hline 2007 & 15 & 8 & 0 & 23 \\
\hline 2006 & 6 & 8 & 5 & 19 \\
\hline 2005 & 10 & 18 & 5 & 33 \\
\hline 2004 & 4 & 10 & 1 & 15 \\
\hline 2003 & 5 & 9 & 0 & 14 \\
\hline 2002 & 3 & 4 & 1 & 8 \\
\hline 2001 & 2 & 6 & 0 & 14 \\
\hline 2000 & 0 & 11 & $\mathbf{8 7}$ & $\mathbf{1 9 6}$ \\
\hline TOTAL & $\mathbf{6 0}$ & & & \\
\hline
\end{tabular}

PHASE VOLTAGE IN MACUSPANA DOS BEFORE AND DURING FASE "B" CVT EXPLOSION MCD-93850-KLV 22-MARZO-2005AT 20:48 HRS

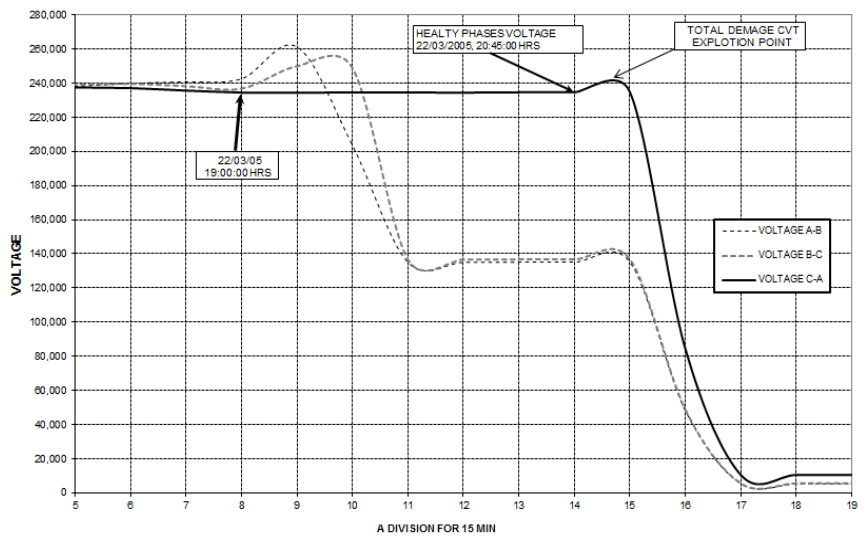

Fig. 4. CCVT fault analysis.

The use of IVTs and fiber optics undoubtedly brings benefits in measurement accuracy and communications, but they also led to several cases of ferroresonance phenomenon. While CCVTs have an anti-ferroresonant element to detune resonance and avoid vibrations, oscillations and eventually explosions, IVTs do not have such element. One reason could be that CFE specification for IVTs does not require that they include an anti-ferroresonance element. For details on CCVT and IVT elements refer to [10]. Two ferroresonance events are discussed in Section IV.

\section{FERRORESONANCE CASE STUDIES}

In recent years the ferroresonance phenomenon had been reported in several electric substations in CFE transmission and sub-transmission systems, see Fig. 5.

Some IVT failures resulted in explosions, affecting circuits and loads connected to the substation and causing severe variations in the frequency and voltage at various points of the CFE system. These variations have been recorded by PMUs, as shown in Figs. 8 and 9. To analyze the ferroresonance 
phenomenon two documented CFE events are presented:

- November 2010 event in the $400 \mathrm{KV}$ gas-insulated substation Tecnologico (TEC),

- January 2011 event in the $400 \mathrm{KV}$ conventional substation Rio Escondido (REC).

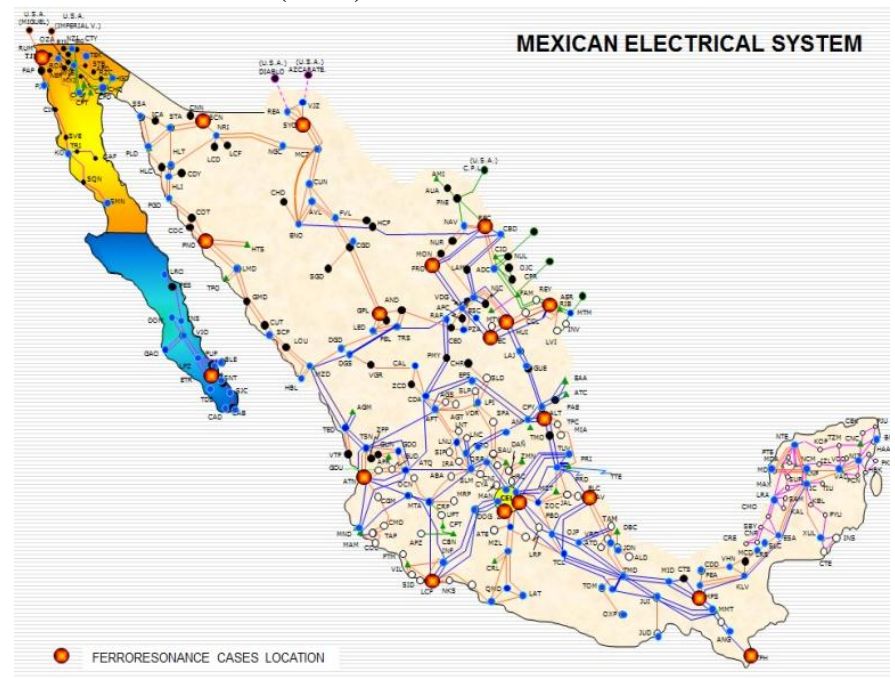

Fig. 5. Ferroresonance cases registered in CFE system.

The second event is further analyzed in Section V which provides calculations for an appropriate reactor to detune or dampen ferroresonance.

\section{A. Case Study 1}

This event took place on November 4th, 2010 in the gasinsulated substation TEC, located in northeast Mexico. As shown on Fig. 6, TEC substation has two $400 \mathrm{KV}$ interconnection lines: TEC-A3750-Huinala Dos (HUO) and TEC-A3H40-Plaza (PZA) and a 400/115 KV 375 MVA transformer $\mathrm{T} 1$.

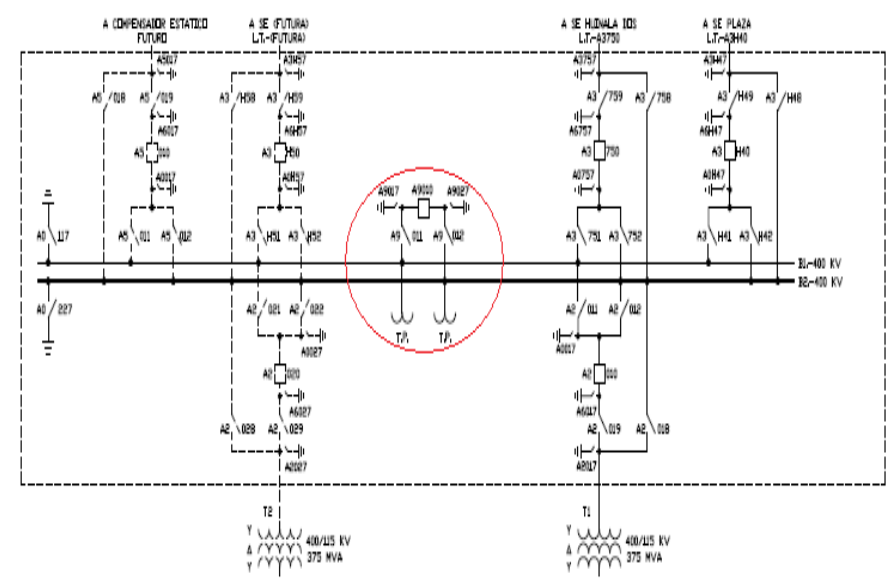

Fig. 6. Gas-insulated substation Tecnológico in $400 \mathrm{KV}$.

On November 4th, 2010 at 15:06 h, maintenance work was performed for breaker TEC-A9010. When all breakers on busbar 2 were open, ferroresonance event occurred and IVT phase B on busbar 2 was damaged (strange noise reported by personnel was caused by IVT core vibration).

On November $4^{\text {th }}, 2010$ at 18:04 hrs., when the circuit breaker TEC-A9010 was put in service (closed), after completed maintenance work (refer to Fig. 7), operated differential protection of busbar 1 (because of IVT damage), and disconnected all devices connected to busbar 1. This caused ferroresonance event that damaged IVT phase B in busbar 1.

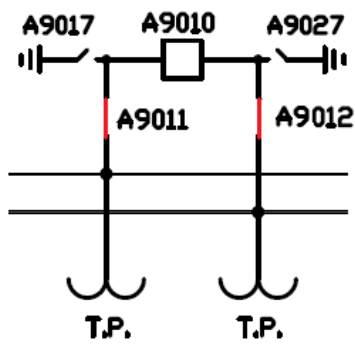

Fig. 7. Switches closed at circuit breaker A9010

After a visual inspection, a differential protection relay 86B-1 was restored, and it was decided to proceed with reconnecting the substation to the CFE network. PMU records of system frequency and voltages at 18:04 h on November 4, 2010 are shown on Fig. 8 and 9.

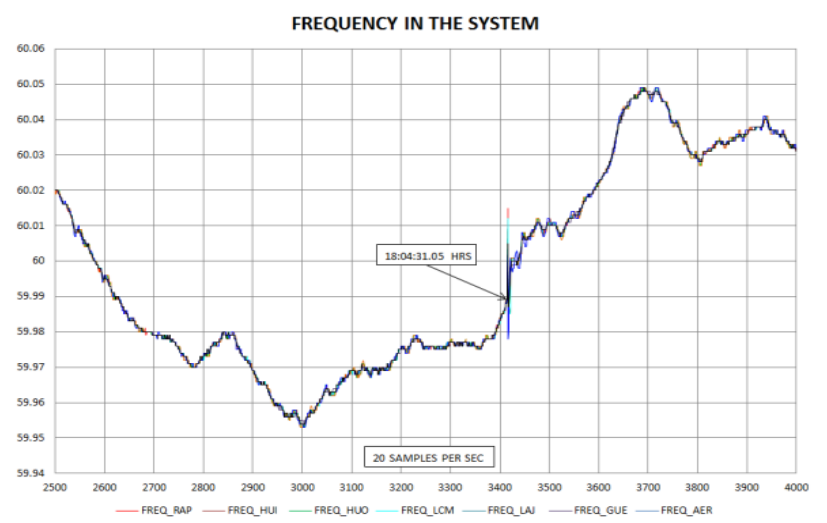

Fig. 8 PMU records of MES frequencies during November 4, 2010 event

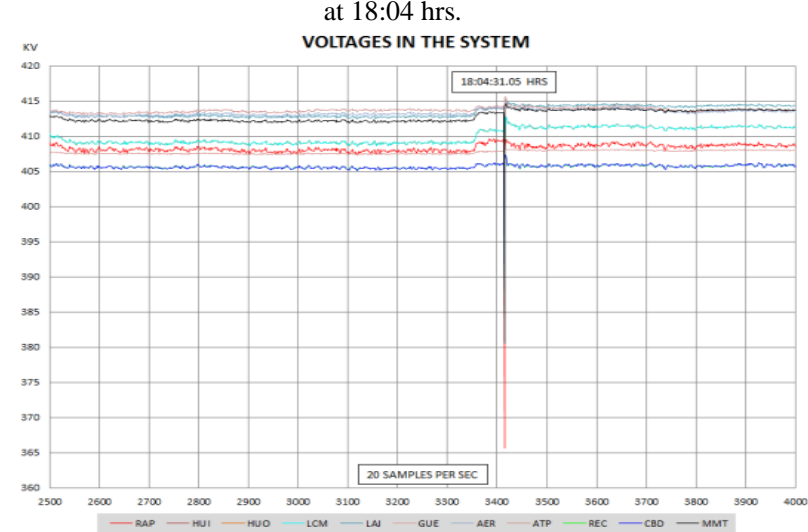

Fig. 9. PMU records of MES voltages during the event on November 4, 2010 at 18:04 hrs.

At 18:27 h busbar 1 in TEC $400 \mathrm{KV}$ was energized through the line PZA-A3H40-TEC. At 18:28 h the circuit breaker PZA-A3H40 was operated due to the fault in the IVT phase B on busbar 1 at $400 \mathrm{KV}$. A second later the circuit breaker TEC-A3750 was closed with the failure still present in the IVT. Then, because the circuit breaker did not clear the fault, the Breaker Failure protection 50 BF operated and disconnected all the equipment tied to busbar 1. Figs. 10 and 
11 show MES frequencies and voltages during this event, provided by PMU records.

To detune ferroresonance in TEC substation at busbar 1 and 2, it was necessary to design and install damping reactors for the $400 \mathrm{KV}$ IVTs.

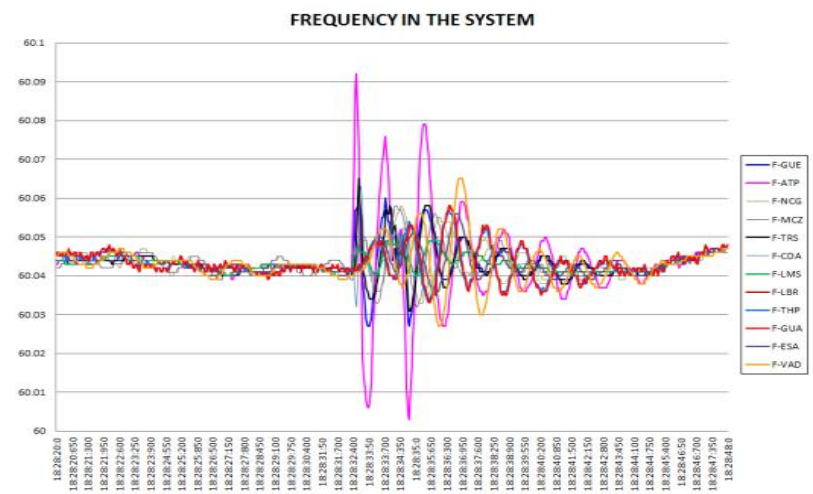

Fig. 10. PMU records of MES frequency during November 4, 2010 event at $18: 28 \mathrm{hrs}$.

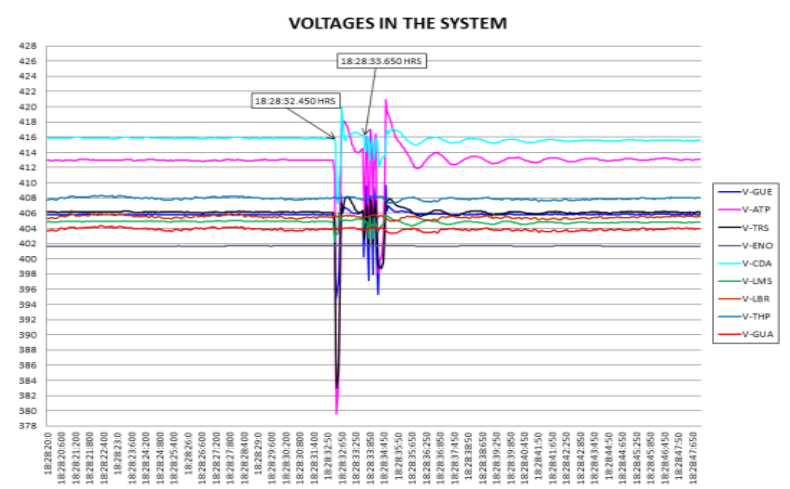

Fig. 11. PMU records of MES voltages during November 4, 2010 event at 18:28 hrs.

\section{B. Case Study 2}

This event documented with relay records, took place on January 8th, 2011 in the conventional $400 \mathrm{KV}$ substation REC, located in northeast Mexico. This event caused damage to IVT phase A at $400 \mathrm{KV}$ due to ferroresonance.

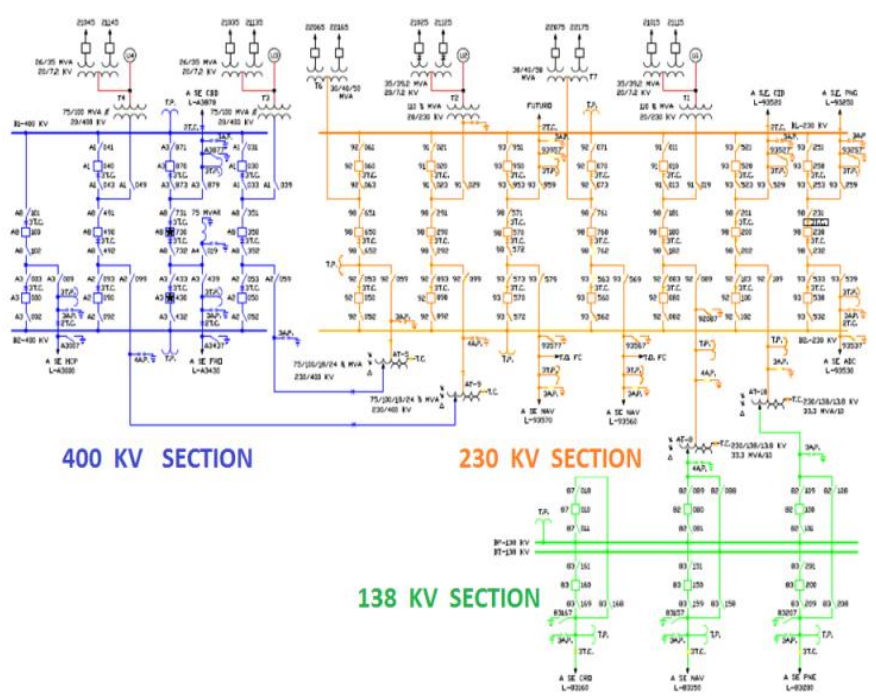

Fig. 12. Conventional substation Rio Escondido diagram.
Substation REC $400 \mathrm{KV}$, as shown on Fig. 12, has two 300 MW generators (U3 and U4), two 400/230 KV 350 MVA auto-transformers and three $400 \mathrm{KV}$ transmission lines: Carbon Dos (CBD), Hercules Potencia (HCP) y Frontera (FRO). It also has one IVT per each phase connected to busbar 1 and busbar 2. The IVTs have $241.500 \mathrm{KV}$ as primary voltage and 115-69 V as secondary voltage. Each circuit breaker has grading capacitance. Location of IVT in REC in shown on Fig. 13.

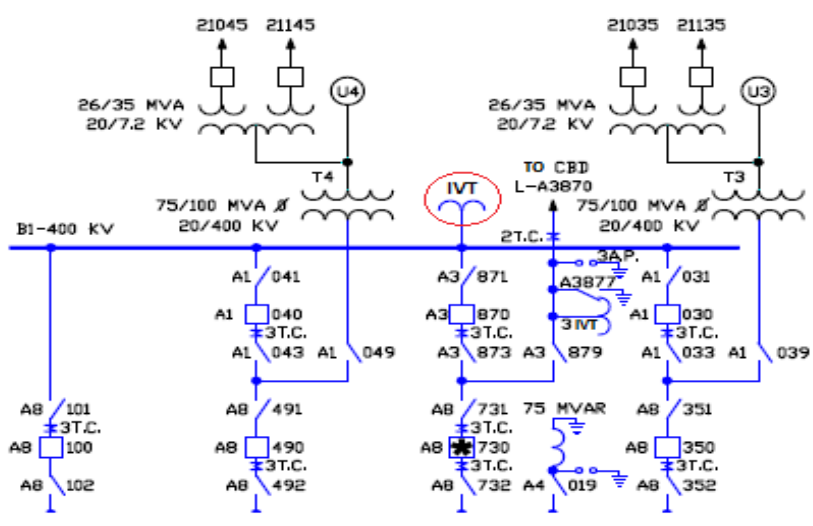

Fig. 13. Location of IVT in REC $400 \mathrm{KV}$.

On January 8th, 2011 at 0:57 h, there was a failure in generator U4 in REC substation. The circuit breaker did not operate to clear the fault, but the breaker failure scheme worked and tripped all equipment connected to busbar 1 . In this moment ferroresonance appeared and IVT phase A was damaged. At 4:34 h, when busbar 1 was restored by closing transmission line REC-Carbon Dos (CBD), the IVT phase A at busbar 1 exploded (because of previous damaged caused by ferroresonance), operated differential protection of busbar 1 . Then ferroresonance in phases B and C occurred. This event was captured by a relay recorder; it is shown in Fig. 14 .

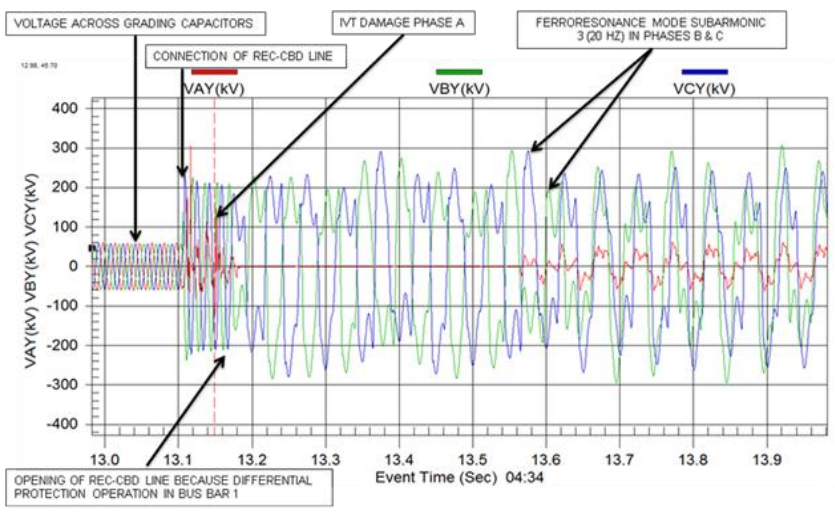

Fig. 14. Disturbance captured in REC on January 8th, 2011 at 4:34 hrs.

On Fig. 14 one can observe the following:

- Pre-fault voltage $(13.0$ - $13.10 \mathrm{sec})$ is provided by voltage applied across the grading capacitance of the circuit breakers, because disconnecting switches were closed.

- At $13.10 \mathrm{sec}$ transmission line CBD was connected to energize REC busbar 1. In this moment IVT phase A was 
damaged.

Fourier analysis was used to determine harmonics present in this event, and a sub harmonic of order 3 or a harmonic of $1 / 3$ was registered.

\section{FERRORESONANCE MITIGATION}

As stated earlier ferroresonance phenomenon can be avoided by disconnecting the applied voltage by a 3-phase interrupting device or by adding an appropriate damping reactor to the circuit. To prevent ferroresonance occurrence a damping reactor should be connected to the secondary winding of the IVT. The reactor should have high impedance under normal operating conditions to eliminate power usage and minimize disturbance of the IVT measurements. Upon ferroresonance occurrence the damping reactor reaches its saturation state earlier than IVT, and becomes a resistor inserted in series that damps the ferroresonance [11].

Parameters of damping reactor depend on the circuit parameters, ferroresonance mode, etc. Calculations of damping reactor parameters and verification of its operation for the Case Study 2 are presented below.

Case Study 2 took place in the $400 \mathrm{KV}$ conventional substation Rio Escondido (REC). To calculate damping reactor parameters and verify its operation, simulations of the REC circuits and event behavior were performed. Equivalent electrical circuit used for REC simulation is shown on Fig. 15. It includes transmissions lines, IVTs for two busbars, circuit breakers with grading capacitance and conductor's capacitance.

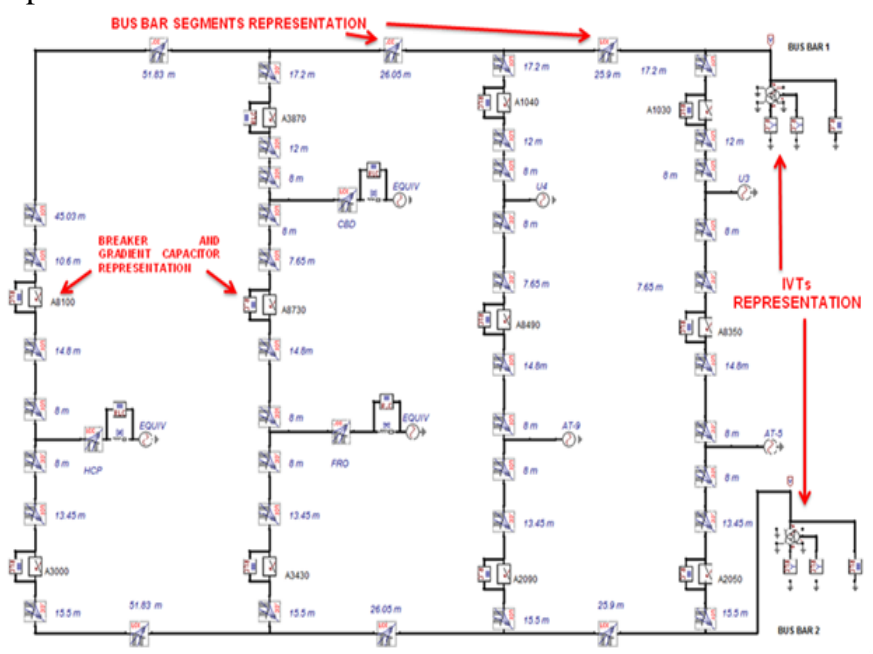

Fig. 15. REC equivalent electrical circuit.

The event which took place in REC on January 8th, 2011 at $4: 34 \mathrm{~h}$ was simulated, and a similar behavior pattern was obtained, see Fig. 16.

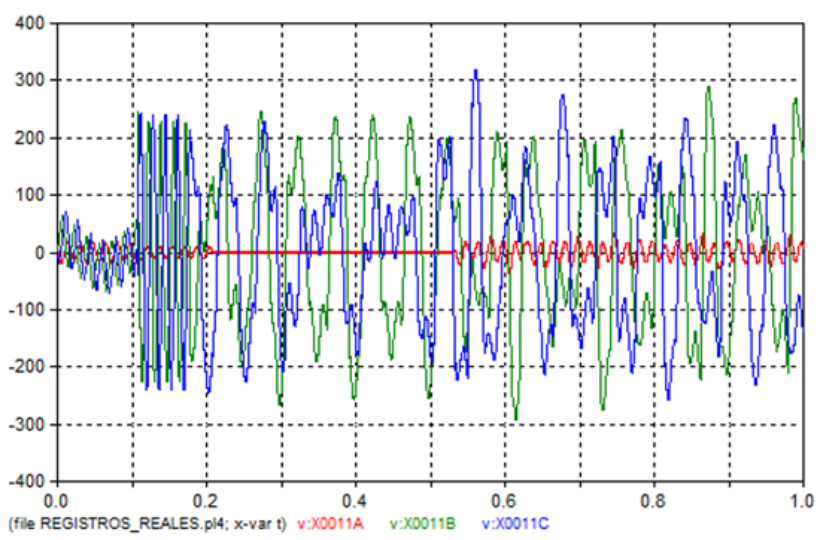

Fig. 16. REC event simulation

Calculations of damping reactor parameters for REC substation sub harmonic 3 ferroresonance mode were made using a method described in [12]. The following was obtained

- Minimum resistance of the damping reactor is $0.11 \mathrm{Ohms}$.

- Operating range of the dumping reactor saturation voltage is $76-162 \mathrm{~V}$.

Verification of the damping reactor operation was performed by simulations using the equivalent electrical circuit shown on Figs. 17 and 18 that includes dumping reactor. For verification the following parameters were used:

- Damping reactor resistance: $0.12 \mathrm{Ohms}$

- Damping reactor saturation voltage: $90 \mathrm{~V}$

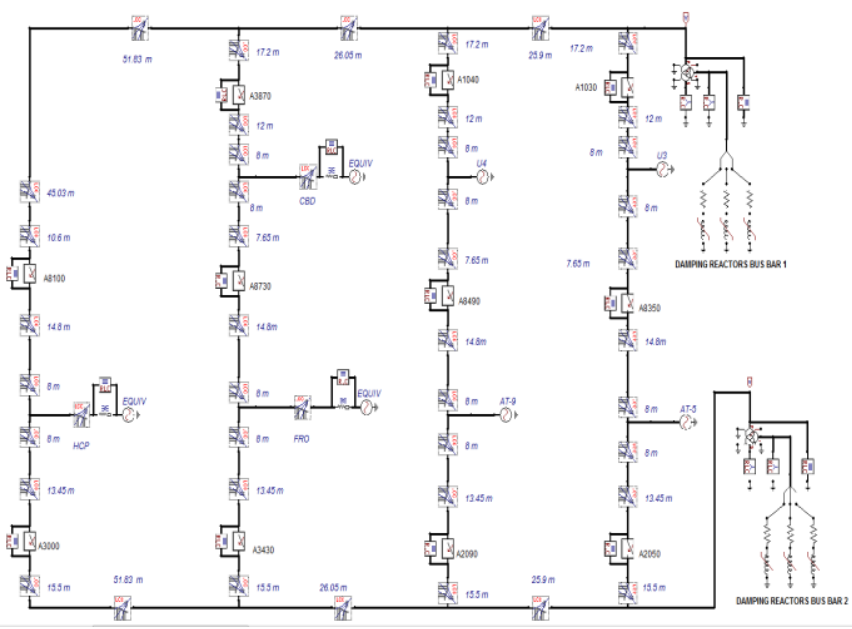

Fig. 17. Equivalent electrical circuit of REC substation with damping reactors.

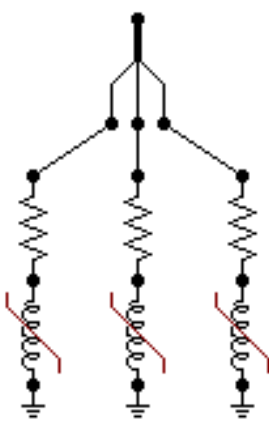

Fig. 18. Damping reactors simulation 
Several operating conditions in the REC substation were simulated to evaluate the damping reactors operation, in particular prevention of the ferroresonance phenomenon.

Simulations of the January 8th, 2011 event in the REC substation, Case Study 2, are shown on Figs.19a and 19b. It is evident that the use of damping reactor allows avoiding the ferroresonant behavior, and therefore can protecting operating personnel, substation equipment, and preventing undesirable energy losses.

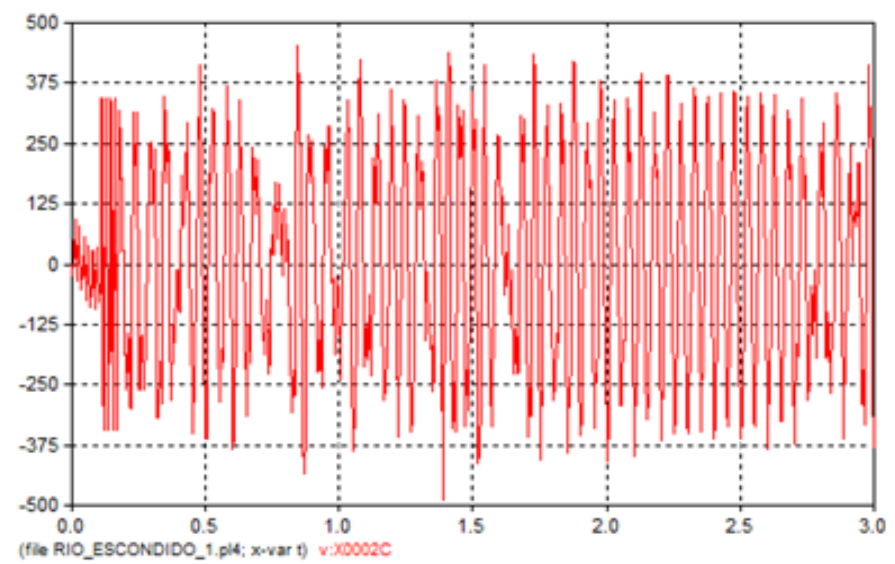

(a) Phase $\mathrm{C}$ simulation without dumping reactor

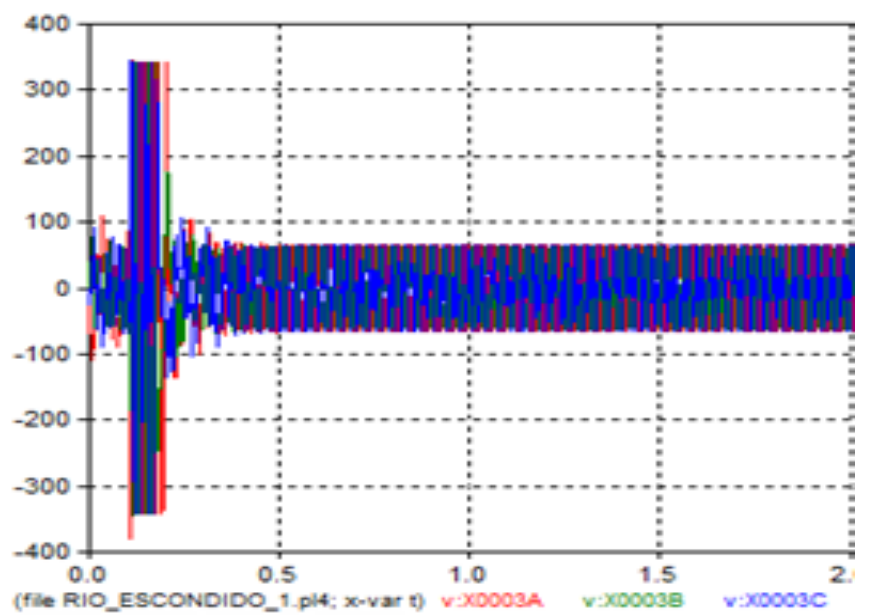

(b) Phases $\mathrm{A}, \mathrm{B}$ and $\mathrm{C}$ simulation with dumping reactor

Fig. 19. Verification of the dumping reactor operation in REC substation.

\section{CONCLUSIONS}

Ferroresonance phenomenon is characterized by the electrical surges and irregular waveforms associated with saturable inductors and capacitors. The capacitance comes from the circuit breakers with gradient capacitors, cable capacitance and capacitance of the busbars. The inductance is provided by the inductive voltage transformers, IVTs.

Due to the replacement of CCVTs by IVTs ferroresonance phenomenon occurs more frequently in the CFE system. Reasons for replacing CCVTs are significant number of their failures, and insufficient measurement accuracy for energy billing. IVT measurements are more accurate but IVTs don't have anti-ferroresonance elements.
Ferroresonance phenomenon can result in very serious damage to the operating personnel and electrical equipment involved, as well as electrical power losses; therefore, it should be understood and avoided. One way to avoid ferroresonance is to use 3-phase interrupting devices to disconnect the applied voltage. Another approach is to add appropriate anti-ferroresonant devices, such as damping reactors to the IVT secondary circuits.

This paper explained the ferroresonance phenomenon and its origins in the CFE systems. Analyses of two Case Studies of the CFE events with ferresonance phenomenon were presented. Damping reactor calculations and verification of its operation are also included.

\section{REFERENCES}

[1] Roger C. Dugan et al, "Electrical Power Systems Quality" Second Edition, McGraw-Hill 2002, ISBN 0-07-138622-X, chapter 4 page 120.

[2] V. Valverde, A.J. Mazón, I. Zamora, G. Buigues. "Ferroresonance in Voltage Transformers: Analysis and Simulations". International Conference on Renewable Energies and Power Quality (ICREPQ'13).

[3] Bethenod, J. (Nov. 30, 1907), "Sur le Transformateur à Résonance", L’Éclairage Électrique 53: 289-96.

[4] Boucherot, P., "Éxistence de Deux Régimes en Ferrorésonance", Rev.Gen. de L'Élec., vol. 8, no. 24, December 11, 1920, pp. 827-828.

[5] Dugan, R. C., "Examples of Ferroresonance in Distribution Systems".

[6] David A. N. Jacobson, Peter W. Lehn and Robert W. Menzies. "Stability Domain Calculations of Period-1 Ferroresonance in a Nonlinear Resonant Circuit". IEEE Transactions On Power Delivery, vol.17, no. 3, July 2002.

[7] Ferracci, Ph., Cahier technique $\mathrm{n}^{\circ}$ 190: "Ferroresonance", Groupe Schneider.

[8] E. D. Price "Voltage Transformer Ferroresonance in Transmission Substations", Conference for Protective Relay Engineers, Texas A\&M University, 1977.

[9] Zhu Xukai, Yang Yihan, Lian Hongbo, Tan Weipu. "Study on Ferroresonance due to Electromagnetic PT In Ungrounded Neutral System". 2004 International Conference on Power System TechnologyPOWER 2004. Singapore, 21-24 November 2004.

[10] ARTECHE. "Inductive Voltage Transformers Oil-Paper Insulated". Up to $525 \mathrm{KV}$.

[11] Kuo-Hsiung Tseng, and Pei-Yu Cheng. "Ferroresonance Experience of 161KV Potential Transformer in Taiwan: Simulation, Measurement and Suppression".

[12] Electrotecnia Arteche hermanos, S.A. "Serial Ferroresonance in voltaje transformers". Adolfo Ibero. ARTECHE, Mungia, España. 